\title{
A Correction Current Injection Method for Power Flow Analysis of Unbalanced Multiple-grounded 4-wire Distribution Networks
}

\author{
Keith Sunderland \\ Technological University Dublin, keith.sunderland@tudublin.ie \\ M. Coppo \\ University of Padova \\ Michael Conlon \\ Technological University Dublin, michael.conlon@tudublin.ie
}

See next page for additional authors

Follow this and additional works at: https://arrow.tudublin.ie/engscheleart2

Part of the Electrical and Computer Engineering Commons

\section{Recommended Citation}

Sunderland, K., Coppo, M., Conlon, M. \& Turri, R. (2017). A correction current injection method for power flow analysis of unbalanced multiple-grounded 4-wire distribution networks. Electric Power Systems Research, 132, pp.30-38. doi:10.1016/j.epsr.2015.10.027

This Article is brought to you for free and open access by the School of Electrical and Electronic Engineering at ARROW@TU Dublin. It has been accepted for inclusion in Articles by an authorized administrator of ARROW@TU Dublin. For more information, please contact arrow.admin@tudublin.ie, aisling.coyne@tudublin.ie, gerard.connolly@tudublin.ie.

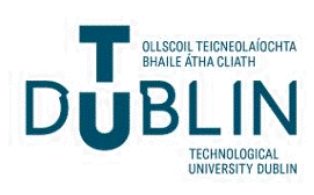




\section{Authors}

Keith Sunderland, M. Coppo, Michael Conlon, and R. Turri

This article is available at ARROW@TU Dublin: https://arrow.tudublin.ie/engscheleart2/184 


\title{
A correction current injection method for power flow analysis of unbalanced multiple-grounded 4-wire distribution networks
}

\author{
K. Sunderland ${ }^{1 *}$, M. Coppo ${ }^{2}$, M. Conlon ${ }^{1}$, R. Turri ${ }^{2}$ \\ ${ }^{1}$ Dublin Institute of Technology, School of Electrical Engineering Systems, Ireland \\ ${ }^{2}$ Department of Industrial Engineering, University of Padova, Italy \\ * Corresponding author
}

\begin{abstract}
Power flow analysis of distribution networks incorporating LV consumer representation needs to be cognizant of an unbalanced load structure and the grounding network between the consumer and network operator (TNC-S earthing). In this paper, the asymmetrical 3-phase (and neutral) power flow problem is solved by a correction current injection methodology applied to a system represented by a complex admittance matrix. The correction current injection technique is adopted to adjust the power exchange of shunt elements, whose nominal admittances are included in the system admittance matrix, through suitable fringing currents in the iteration process. This methodology offers an improved and more robust alternative for asymmetrical network scenarios under unbalanced power flow conditions when compared to the standard power flow methodologies, such as the Newton-Raphson or the forward-backward sweep approaches. These well-known methods may encounter convergence issues as a consequence of the specific consumer/network earthing arrangements especially when they need to be defined throughout the network. The algorithm presented here has been applied to a 4-wire representation of a suburban distribution network within Dublin city,
\end{abstract}


Ireland, which incorporates consumer connections at single-phase (230V-N). The analysis presented uses the correction-current injection power flow algorithm in conjunction with the network model to consider the impact of distributed wind and solar (PV) generation systems ( $D w G$ and $D p v G$ respectively), for a range of load profiles. Keywords:

Distribution Network analysis, power flow, urban environments, small wind turbines, small solar PV systems, admittance network correction, fringe current correction.

\section{Introduction}

More than half the World's population lives in urban areas, occupying less than $3 \%$ of the Earth's ice-free land area. Cities are responsible for between $71 \%$ and $76 \%$ of $\mathrm{CO}_{2}$ emissions from global final energy use [1], much of it derived from fossil-fuel based electricity generation. Moving towards a more sustainable economy, urban areas need to develop smart energy networks that can both generate and deliver renewable electricity in a predictable and consistent manner. Significant momentum is being achieved in economic "greening" and in 2011 alone, renewable energy sources accounted for 44\% of new generation added worldwide [2]. While the majority of this new capacity comes from larger plants (such as wind farms), the influence of the residential sector should not be underestimated and in countries such as the UK, significant efforts are being made to capture this market $[3,4]$. The residential portion of total energy use accounts for $32.79 \%$ [5] and 30.9\% [6] in the US and Euro zone respectively. The connection of small and micro-generation at consumer level could contribute positively towards national renewable energy targets; particularly in a smart grid context. This kind of evolution requires a more integrated, distributed and bi-directional energy supply chain, which is representing a tough challenge for distribution network operators. These 
networks were originally designed for a vertically integrated power system with several large power plants and a mainly passive grid. The presence of generation units in distribution networks leads to the need for a detailed modeling of those systems with a particular focus on the LV grid which is generally an asymmetrical network with unbalanced loading conditions on the three phases. The power flow calculation is used to compute the steady state operating condition of a power system and its solution should be fast, require low storage requirements and be reliable and versatile through an inherent simplicity [7, 8]. The algorithms generally adopted are Gauss-Seidel or Newton-Raphson (and its decoupled versions [9]) which are sufficiently robust and fast even for large networks but don't allow a very easy extension to a multi-phase system. This aspect can be neglected when considering transmission systems (considering the single-phase equivalent circuit) but it could assume an important role when unbalanced load and generation scenarios are involved, as in distribution networks. In a recent review of Power Flow studies, Balamurugan and Srinivasan [10] describe how threephase power flow analysis can be considered in terms of two different reference frames, namely the phase frame and the sequence frame. The phase frame, incorporates methods such as forward/backward sweep, (Kirchoff) compensation, implicit ZBus Gauss method and modified Newton/Newton-like methods. They all consider unbalanced quantities directly. On the other hand, the sequence frame employs decoupled positive, negative and zero sequence networks to represent the unbalanced three-phase system and to solve the unbalanced three-phase power flow. The multi-conductor correctioncurrent injection power flow methodology presented in this paper uses a phase frame reference. All the network elements are represented through suitable admittances in order to result into a system's admittance matrix including all the network's phases. A 
similar approach seems to be used by the OpenDSS software released by EPRI [11, 12], which also employs a phase frame of reference for the solution of the power flow problem in generic n-phase networks.

This paper provides a detailed description of the asymmetrical $n$-phase power flow solution presented in [10], which is based on a complex admittance matrix methodology [13] to consider a representative urban distribution network [8, 14]. The system admittance matrix is obtained through the definition of self and mutual couplings among the phases in order to allow the representation of any number of phase and earth conductors (e.g. neutral conductor, earth wires and shields). In the iterative power flow calculation loop a "Fringing" Correction Current (FCC) methodology is adopted to include the required voltage dependency of shunt elements through a suitable correction current injection in parallel to load/generation rated admittance(s). The power flow algorithm (FCC) facilitates balanced and unbalanced distribution system solutions, which can be radial or meshed. Furthermore, the algorithm is sufficiently flexible to allow considering n-phase line configurations. In this paper it has been applied to a 3phase/4-wires LV real network considering also the systematic earthing along the lines (TN-CS). The aim of the paper is to provide a thorough description of the correctioncurrent injection power flow methodology, giving a detailed description of how to model the elements and to demonstrate its applicability on a section of Irish active LV distribution network under unbalanced operating conditions. A comparison of the results obtained from the proposed methodology and the software OpenDSS and PowerFactory is reported to validate the results.

\section{Asymmetrical Power Flow Method}


In this section a description of the algorithm adopted for the asymmetrical power flow analysis is provided. This correction-current injection algorithm evolved from the complex admittance matrix power flow methodology described in [13] by including a multi-conductor network structure in order to consider any number of phase and earth conductors. The main feature of this method is the inherent flexibility in how multiconductor network models and their associated effects are considered. Mutual coupling influences between phases, are computed through a method that was originally developed for calculating electromagnetic coupling of complex conductor geometries [15]. The use of such a multi-conductor approach facilitates accounting for any kind of interaction between phases meaning that any network shunt element connections can be considered in terms of the system's phase and reference potentials and with respect to specific grounding (earthing) options. This feature intrinsically allows any generic network with asymmetrical structure and operating under unbalanced conditions to be considered.

\section{Figure 1}

Figure 1 shows a typical distribution system, incorporating network structure, load, generation and grounding elements. The branch element admittance matrix is composed through an incidence matrix approach [16] computing the mutual admittances between the system's buses, which are represented as n-phase ports ("nodes"). The network shunt elements and grounding admittances are connected to these nodes, providing a linkage between the phase potentials and the system ground.

\subsection{Branch Elements}

Branch elements are included in the network admittance matrix by considering a nphase $\pi$-model. Each branch admittance matrix is comprised of longitudinal impedance 
$\mathbf{Z}$ and transversal admittance $\mathbf{Y}_{\mathbf{t}}$ matrices as described in equation (1) and illustrated in Figure 2:

$\mathbf{Y}_{\text {Branch }}=\left[\begin{array}{cc}\mathbf{Z}^{-1}+\frac{\mathbf{Y}_{\mathbf{t}}}{2} & -\mathbf{Z}^{-1} \\ -\mathbf{Z}^{-1} & \mathbf{Z}^{-1}+\frac{\mathbf{Y}_{\mathbf{t}}}{2}\end{array}\right]$

\section{Figure 2.}

The so-called $\mathbf{Y}_{\text {Branch represents the relationship between currents (positive if entering) }}$ and voltages (with respect to a common zero-voltage reference) of the $2 n$ ports of the branch element. The construction of the $\mathbf{Z}$ and $\mathbf{Y}_{\mathbf{t}}$ sub-matrices within the $\pi$-model is obtained using the classical Carson-Clem formulation for a n-phase branch as described in [15]. An approximation of the correction terms for the real and imaginary components of the external part of the self and mutual impedance with earth return, is also provided in [15]. It is important to note that in practical cases these correction terms could be the dominant impedance in the 4-wire model, especially when considering unbalanced operation. The longitudinal impedance matrix $\mathbf{Z}$ contains the self and mutual impedances for each phase. Given two circuits $i$ and $j$ those terms are calculated as in equations (2) and (3):

$$
\begin{aligned}
& Z_{i i}=R_{i}+R_{e}+j \omega \cdot 2 \cdot 10^{-4} \cdot \ln \left(\frac{D_{e}}{r_{i}}\right)\left[\frac{\Omega}{k m}\right] \\
& Z_{i j}=R_{e}+j \omega \cdot 2 \cdot 10^{-4} \cdot \ln \left(\frac{D_{e}}{d_{i j}}\right)\left[\frac{\Omega}{k m}\right]
\end{aligned}
$$

where

$$
\begin{aligned}
& \mathrm{R}_{\mathrm{i}}: \text { DC resistance }[\Omega / \mathrm{km}] \\
& \mathrm{r}_{\mathrm{i}}: \quad \text { phase conductor radius }[\mathrm{m}] \\
& \mathrm{d}_{\mathrm{ij}}: \text { mutual distance between conductors } \mathrm{i} \text { and } \mathrm{j}[\mathrm{m}] .
\end{aligned}
$$


In equations (2) and (3), the hypothesis of soil finite conductivity is duly considered by an earth return path with depth $D_{e}$ and resistance $R_{e}$ [15], as defined in equations (4) and

(5) below:

$$
\begin{aligned}
& R_{e}=\pi^{2} f \cdot 10^{-4}\left[\frac{\Omega}{k m}\right] \\
& D_{e}=659 \sqrt{\frac{\rho}{f}} \quad[m]
\end{aligned}
$$

where

$$
\begin{aligned}
& \text { f: system frequency }[\mathrm{Hz}] \text {; } \\
& \rho: \text { soil conductivity }[\Omega \mathrm{m}] \text { (typically } 100 \Omega \mathrm{m} \text { ). }
\end{aligned}
$$

The transversal admittance matrix $\mathbf{Y}_{\mathbf{t}}$ represents the capacitive self and mutual susceptances, as evaluated through the Maxwell's potential coefficients. For the power flow problem in LV networks however, these terms have only a marginal effect. Once YBranch is computed for each branch element, the system's nodal admittance matrix can be easily constructed through an incidence matrix that defines the topology of the network.. For an m-bus, n-conductors network, $Y_{\text {network }}$ is a $(m \times n) \times(m \times n)$ square matrix.

\subsection{Shunt Elements}

Loads, generators and in general any shunt element, can be represented by a combination of a constant shunt admittance as calculated in (6) and, if needed, through suitable correction current injector as highlighted in Figure 1.

For a single-phase shunt element connected between nodes $k$ and $h$, the nominal complex admittance can be calculated as follows: 
$Y_{k h}=\frac{S_{k h(0)}^{*}}{\left|U_{k h(0)}\right|^{2}}, \quad\left(U_{k h}=E_{k}-E_{h}\right)$

where $S_{k h(0)}$ is the rated power of the shunt element and $E$ are the potentials of the $k$ and $h$ nodes. The subscript $(0)$ indicates that the values are referred to the initial guess (nominal values).

This kind of approach enables the inclusion of the shunt elements within the system admittance matrix allowing the voltage dependency to be customised according to the chosen model.

The shunt element apparent power can be described through equation (7), which refers to the $i$-th iteration. Separating the constant admittance facilitates a definition of a correction current vector $\Delta I$, which allows the introduction of a specific voltage dependency without changing the shunt element's admittance. In this way, the shunt element nominal admittance can be included in the system admittance matrix, which is then composed entirely of constant values.

$$
S_{k h(i)}^{*}=Y_{k h} \cdot\left|U_{k h(i)}\right|^{2}-U_{k h(i)} \Delta U_{k h(i)}
$$

In the following, equation (7) is considered in terms of the ZIP model in order to clarify the concept of the aforementioned correction current approach.

$$
S_{k h(i)}^{*}=S_{k h(0)}^{*} \cdot\left[\left(\frac{\left|U_{k h(i)}\right|}{\left|U_{k h(0)}\right|}\right)^{2} \cdot k_{Z}+\left(\frac{\left|U_{k h(i)}\right|}{\left|U_{k h(0)}\right|}\right) \cdot k_{I}+k_{P}\right], k_{z}+k_{l}+k_{p}=1
$$

With regard to the constant impedance part, the $\Delta I$ component in equation (7) is set to zero. For the constant current part of the ZIP model in equation (8), which describes the linear voltage dependency, the correction current $\Delta I$ can be derived as follows: 


$$
\begin{aligned}
& S_{k h(i) I \%}^{*}=k_{I}\left(\frac{\left|U_{k h(i)}\right|}{\left|U_{k h(0)}\right|}\right) \cdot Y_{k h} \cdot\left|U_{k h(0)}\right|^{2}=k_{I}\left(Y_{k h} \cdot\left|U_{k h(i)}\right|^{2}-U_{k h(i)} \Delta I_{k h(i)} I \%\right) \\
& \Delta I_{k h(i) I \%}=k_{I} \cdot \frac{Y_{k h}}{U_{k h(i)}}\left(\left|U_{k h}\right|_{(i)}^{2}-\left|U_{k h}\right|_{(i)}\left|U_{k h}\right|_{(0)}\right)
\end{aligned}
$$

The constant power share may also be expressed through a suitable correction current as described in equations (11-12):

$$
\begin{aligned}
& S^{*}{ }_{k h(i)}{ }_{P \%}=k_{P} \cdot Y_{k h} \cdot\left|U_{k h(0)}\right|^{2}=k_{P}\left(Y_{k h} \cdot\left|U_{k h(i)}\right|^{2}-U_{k h(i)} \Delta I_{k h(i)} P \%\right) \\
& \Delta I_{k h(i) P \%}=k_{P} \cdot \frac{Y_{k h}}{U_{k h(i)}}\left(\left|U_{k h}\right|_{(i)}^{2}-\left|U_{k h}\right|_{(0)}^{2}\right)
\end{aligned}
$$

Finally, equation (13) describes the composition of the ZIP model, with an emphasis on the fact that the different voltage dependency shares may be represented by separate current injectors.

$$
S^{*}{ }_{(i)}=Y_{k h} \cdot\left|U_{k h(i)}\right|^{2}-\left(\Delta I_{k h(i)} I \%+\Delta I_{k h(i)} P \%\right) \cdot U_{k h(i)}
$$

\subsection{Bus nodes connections and grounding}

One of the most important features of this kind of power flow calculation is that it allows to customize the connection between network phases and ground at any point of the system. This allows for a consideration of the specific connection layouts and grounding options without the necessity of utilizing the sequences approach.

As shown in Figure 1, the system is composed by simply including admittances for each coupling among conductors. The same approach is applicable for the connection between neutral and ground (green elements in Figure 1), which can therefore be represented as a self-admittance at the grounded bus.

\subsection{Power Flow solution methodology}


The power flow solution is based on the complex admittance matrix approach developed for balanced systems modeled as single-phase equivalents [13]. After computing the branch and shunt elements matrix as described previously, the system admittance matrix is composed as illustrated in Figure 1. The $\mathbf{Y}_{\text {network }}$ matrix is obtained by including the branch element sub-matrices through a suitable topology matrix. The constant admittance part of the shunt elements is also added to the network matrix as shown in Figure 3, so at that point the system buses are only represented by ports where the correction currents calculated as in 2.2 can be updated.

\section{Figure 3}

An important point to note, is that the inclusion of admittances representing the shunt elements in the system admittance matrix can reduce power flow inversion problems, as it is inverted only once by the Fringe-Currents Injection technique [13].

At this stage, a direct solution is iterated updating the shunt currents, through the system showed in Figure 4. The approach specifically isolates the effects of the slack bus (imposed voltage vector EsL) from the other shunt elements, so that it can be described as in (14) and (15):

\section{Figure 4}

$$
\begin{aligned}
& \mathbf{I}_{\mathbf{S L}}=\mathbf{Y}_{\mathrm{SL}-\mathrm{SL}} \mathbf{E}_{\mathbf{S L}}+\mathbf{Y}_{\mathrm{SL}-\mathbf{S H}_{\mathbf{2}} \mathbf{E}_{2-\mathrm{m}}}, \\
& \Delta \mathbf{I}_{2-\mathrm{m}}=\mathbf{Y}_{\mathrm{SH}-\mathrm{SL}} \mathbf{E}_{\mathrm{SL}}+\mathbf{Y}_{\mathrm{SH}-\mathrm{SH}} \mathbf{E}_{2-\mathrm{m}}
\end{aligned}
$$

\subsection{Iterative loop}

An iterative loop is applied to find a solution for the system represented in (14) and (15). Equation (16) shows how the $\mathbf{E}_{\mathbf{k}} \ldots \mathbf{E}_{\mathbf{h}}$ potentials may be related to the $\Delta \mathbf{I}$ currents 
$\mathbf{E}_{2-\mathbf{m}}=\mathbf{Y}_{\mathbf{S H}-\mathbf{S H}}^{-1}\left(\Delta \mathbf{I}_{2-\mathbf{m}}-\mathbf{Y}_{\mathbf{S H}-\mathbf{S L}} \mathbf{E}_{\mathbf{S L}}\right)$

The initial condition is evaluated by zeroing the $\Delta \mathbf{I}$ array. An iterative loop subsequently updates these correction current terms as the potentials $\mathbf{E}_{\mathbf{k}-\mathbf{h}}$ change. Convergence is obtained when the voltage magnitude and phase angle changes remain below given thresholds. For a generic $i$-th iteration, convergence is reached if the conditions (17) and (18) are verified:

$$
\begin{aligned}
& \left|\mathbf{E}_{(\mathbf{i}+\mathbf{1})}\right|-\left|\mathbf{E}_{(\mathbf{i})}\right| \leq \varepsilon_{M} \\
& \angle \mathbf{E}_{(\mathbf{i}+\mathbf{1})}-\angle \mathbf{E}_{(\mathbf{i})} \leq \varepsilon_{A}
\end{aligned}
$$

where $\varepsilon_{M}$ and $\varepsilon_{A}$ are the magnitude and angle thresholds respectively. It should be noted that in using this approach, the $\mathbf{Y}$ matrix does not need to be updated, so the inversion in (16) is not repeated during the iterative solution. Furthermore, this method allows the consideration of different kinds of shunt elements (loads or generators) by simply adapting the injected currents $\Delta \mathbf{I}$.

\section{Network Model}

The network considered in this work is depicted in Figure 5. It consists of a section of LV (urban) distribution network incorporating 74 households facilitated by 10 minipillar connections (along the LV feeder) and supplied by a 10/0.4 kV supply.

\section{Figure 5}

The network is radial in structure with the sub-distribution branch sections being facilitated through 'mini-pillars' (hereafter, referred to as pillars). Each pillar (busbars from B to J in Figure 5) accommodates single-phase consumer connections (domestic installations), each with distinct earthing provision (TN-C-S). Service cabling, from pillars to consumers is $25 / 16 \mathrm{~mm}^{2}$ concentric neutral. The cabling from the substation 
transformer to the first pillar (and each pillar thereafter) is either $185 / 70 \mathrm{~mm}^{2}$ crosslinked polyethylene (XLPE) or $70 \mathrm{~mm}^{2}$ paper-insulated (NAKBA). Figure 6 illustrates the network structure from the transformer down to the consumer in context with the pillar/consumer earthing provision. The earth electrode impedances are modeled as $5 \Omega$ resistances at customers connections and $1 \Omega$ resistances at the pillars.

\section{Figure 6}

The supply voltage at the $10 / 0.4 \mathrm{kV}$ transformer operates in accordance with the assumed maximum voltage drop limits as defined in the EN50160 voltage standard [17]. Essentially, the DNO is prescribed to deliver electricity in a voltage range of $207 \mathrm{~V}$ to $253 \mathrm{~V}\left( \pm 10 \% \mathrm{~V}_{\text {Nominal }}\right.$ of $\left.230 \mathrm{~V}\right)$. In the analysis presented here, the sending voltage at the feeder bus is $+5 \%$ with respect to the nominal voltage.

The passive network model parameters and associated data were supplied by the Irish DNO, ESB Networks [14]. The analysis presented in section 4 considers a scenario with generation units connected to the households (wind generation, $(D w G)$ and solar PV $(\operatorname{Dpv} G))$.

\subsection{Cable Modeling}

The generic cable considerations (including geometric mean radii and relative conductor distances) are modelled in accordance with Kersting's approximations [18] applied through the methodology described in section 2.1 starting from the Carson-Clem formulae.

Figure 5 shows that there are three sub-distribution cable types employed, however in the analysis presented in section 4 , the same cable, namely the XLPE $\left(180 \mathrm{~mm}^{2}\right)$, is used throughout the network branch connections. The branch cable lengths are reported explicitly in Figure 5. For all the connections between consumers and pillars, the cable 
lengths are normalized to the same length, $31.5 \mathrm{~m}$, which is actually the average pillar to consumer length associated for the actual network.

The branch impedance matrix is built from the self and mutual terms of the $\pi$-model shown in Figure 2. Each branch of the main feeder connecting the pillars is represented as a full $4 x 4$ matrix, while the single-phase connections are modeled according to equation (19), depending on which phase the customer is connected to.

$\mathbf{Z}_{\mathbf{a n}}=\left[\begin{array}{cccc}Z_{i i} & 0 & 0 & Z_{i j} \\ 0 & 0 & 0 & 0 \\ 0 & 0 & 0 & 0 \\ Z_{j i} & 0 & 0 & Z_{j j}\end{array}\right] ; \mathbf{Z}_{\mathbf{b n}}=\left[\begin{array}{cccc}0 & 0 & 0 & 0 \\ 0 & Z_{i i} & 0 & Z_{i j} \\ 0 & 0 & 0 & 0 \\ 0 & Z_{j i} & 0 & Z_{j j}\end{array}\right] ; \mathbf{Z}_{\mathbf{c n}}=\left[\begin{array}{cccc}0 & 0 & 0 & 0 \\ 0 & 0 & 0 & 0 \\ 0 & 0 & Z_{i i} & Z_{i j} \\ 0 & 0 & Z_{j i} & Z_{i j}\end{array}\right]$

The full $4 x 4$ description of the XLPE $\left(180 \mathrm{~mm}^{2}\right)$ cable is presented in equation (20).

$\mathbf{Z}_{\mathbf{a b c n}}=\left[\begin{array}{cccc}26.13+74.97 i & 4.93+68.73 i & 4.93+68.73 i & 4.93+66.55 i \\ 4.93+68.73 i & 26.13+74.97 i & 4.93+66.55 i & 4.93+68.73 i \\ 4.93+68.73 i & 4.93+66.55 i & 26.13+74.97 i & 4.93+68.73 i \\ 4.93+66.55 i & 4.93+68.73 i & 4.93+68.73 i & 26.13+74.97 i\end{array}\right] \times 10^{-5} \Omega / \mathrm{m}$

\subsection{Distributed Generators Modelling}

Micro generation in Ireland is defined as a technology that can deliver $25 \mathrm{~A}$ at $230 \mathrm{~V}$ or $16 \mathrm{~A}$ at $400 \mathrm{~V}$ [19], as for the associated technical guidelines published by ESB Networks (Irish DNO) [17]. For this paper, micro wind generation $(D w G)$ and solar PV $(D p v G)$ generation systems are connected to specific consumers as illustrated in Figure 5.

The $D w G$ units are modeled by decomposing the power curve associated with a commercially available $2.5 \mathrm{~kW}$ wind turbine into a $10^{\text {th }}$ order polynomial equation [20]. 
The urban wind speed reference employed here is based on the analysis presented in [21] and the wind generation is considered to be installed in suburban locations with a turbine nacelle height of $12 \mathrm{~m}$.

The $D p v G$ units' power output is calculated according to the approach presented in [22], which employs a cell efficiency based on cell temperature and solar irradiance. The cell efficiency is calculated starting from a reference efficiency (18\% referring to a $1 \mathrm{~kW}$ panel output) and is subsequently adjusted according to the temperature variation (through a coefficient of efficiency decline with temperature). For this paper, $2 \mathrm{kWp}$ panels are considered for customers with $D p v G$ installations. Meteorological observations of solar insolation and temperature within Dublin city are employed for this research.

\subsubsection{Loads Modelling}

The loads have been characterized by time-varying power profiles deduced from a representative year data expressed in hourly interval profiles [23]. Consumer hourly demand is based on an annual consumption of electricity of 5000kWh [24], which represents an average hourly demand of $0.571 \mathrm{~kW}$.

\section{Analysis}

The network reaction to variable load/generation over a 24-hour period is analyzed by mixing the three power profiles shown in Figure 7 for the loads, referring to different seasons (load 1, 2 and 3 representing Autumn, Winter and Spring respectively). Regarding the $D w G$ and $D p v G$ models, the profiles shown in Figure 7 result from meteorological data (wind speed, solar irradiance and temperature) as recorded over a 24-hour periodin Autumn, used as input parameters in calculating their power output 
over the same period. Both generators and loads in this analysis have been modeled as constant power units with the power values changing according to the respective profiles and meteorological data. So in respect to the ZIP model description presented in equations (8-13), the value of the parameter $k_{P}$ is 1 .

\section{Figure 7}

Table 1 illustrates the load/generation variation statistics over the respective $24 \mathrm{hr}$ periods with Autumn being chosen for the specific generation considerations.

\section{Table 1}

Initially $12 \mathrm{pm}$ is highlighted for consideration. In this regard, the different load profiles identified in Figure 7 lead to total power consumptions of $0.469 \mathrm{~kW}, 0.479 \mathrm{~kW}$ and $0.806 \mathrm{~kW}$ (unity power factor) at the assigned consumer connections (as illustrated in Figure 5). The total amounts of power produced by $D w G$ and $D p v G$ (as assigned to consumer connections in Figure 5) are respectively $0.398 \mathrm{~kW}$ and $0.475 \mathrm{~kW}$ with 0.95 power factor being applicable to both DG types. Table 2 provides the results in terms of currents at the PCC and busbar voltages, reporting detailed results for Pillars B and J as the extreme ends of the network. The same analysis has been conducted using software OpenDSS and DigSilent PowerFactory and the results reported in Table 3 validate the corresponding results obtained through the proposed methodology.

\section{Table 2}

\section{Table 3}

The voltage profile along the feeder on the 24-hours period is shown in Figure 8 (a) for each phase and the neutral conductor employing the same presentation logic used in 
Table 2. Figure 8 (b) illustrates the voltage unbalance as experienced at the respective pillars, given as the ratio between the negative and positive sequences in percent [25].

\section{Figure 8}

The maximum voltage drop along the feeder can be quantified as about $2 \%$ of the nominal voltage, being similar in each phase, while the pillar voltage unbalance varies from $0.002 \%$ to $0.198 \%$ and is within the acceptable tolerance (2\%). This is mainly due to the distributed neutral grounding throughout the grid (including customer buses) and to the relatively low power flow values, as derived from the reference scenario considered for loads and generators.

The voltage statistics for each phase over the 24-hour period are provided in Table 3, which shows a comparison of the active network case (leading to the results in Figure 8) to the passive network case. The results show that the active network scenario leads to higher values in all the statistical categories even if within an acceptable tolerance. Table 3

\section{Conclusions}

The paper presents a detailed description of an asymmetrical power flow algorithm based on the correction-currents injection approach. The algorithm is quite flexible and allows virtually any number of phase and neutral conductors to be considered. The innovation in the solution approach is provided by the injection of correction currents in parallel to the nominal admittances of the shunt elements. This allows to represent any kind of voltage dependency, resulting in a power output adjustment that involves both loads and generators. Another important feature of this power flow algorithm is the possibility to set different grounding options for each network bus. This aspect could be crucial when dealing with specific requirements as in the case of the Irish LV system, 
where neutral conductors are grounded in different sections of the same network. In this paper, an Irish real LV network is modeled in detail in order to show the potential and suitability of the algorithm to simulate unbalanced and asymmetrical networks. Whereas the load/generation considered in this work are hourly averages, future work will consider higher resolution data and different consumer demographics. Short term demand can vary significantly with high level peak demand and such fluctuations should be considered in terms of network tolerances and generation connection growing. Further research should be carried out to consider the possible effects derived from different earthing conventions from both the supply and the customer perspectives. This work could ultimately consider the effects of different earthing approaches in managing the neutral conductor in low voltage networks .

\section{Aknowledgements}

The authors would like to thank Mr. Anthony Walshe of ESB Networks for his help and cooperation in providing the distribution network parameters.

\section{References}

[1] K. C. Seto, S. Dhakal, A. Bigio, H. Blanco, G.C. Delgado, D. Dewar, L. Huang, A. Inaba, A. Kansal, S. Lwasa, J.E. McMahon, D.B. Müller, J. Murakami, H. Nagendra, A. Ramaswami, "Human Settlements, Infrastructure and Spatial Planning. Contribution of Working Group III to the Fifth Assessment Report of the Intergovernmental Panel on Climate Change," 2014.

[2] A. McCrone, "Global trends in renewable energy investment," Frankfurt School of Finance \& Management,2012.

[3] AEA. (2011, 12th July, 2012). The AEA Microgeneration Index

[4] DECC, "Microgeneration Strategy," Department of Energy and Climate Change2011. 
[5] EIA. (2012, 24/02/13). Annual Energy Review [on-line,]. Available: http://www.eia.gov/totalenergy/data/annual/pdf/aer.pdf

[6] EEA. (2012, 24/02/13). Final electricity consumption by sector, EU-27 [online]. Available: http://www.eea.europa.eu/data-and-maps/figures/finalelectricity-consumption-by-sector-4

[7] S. F. Mekhamer, S. A.Soliman, M.A. Moustafa, M.E. El-Hawary, "Load flow solution of radial distribution feeders: a new contribution," International Journal of Electrical Power \& Energy Systems, vol. 24, pp. 701-707, 2002.

[8] K. M. Sunderland and M. F. Conlon, "4-Wire load flow analysis of a representative urban network incoprating SSEG," in Universities Power Engineering Conference (UPEC), 2012 47th International, 2012, pp. 1-6.

[9] B. Stott, "Review of load-flow calculation methods," Proceedings of the IEEE, vol. 62, pp. 916-929, 1974.

[10] K. Balamurugan and D. Srinivasan, "Review of power flow studies on distribution network with distributed generation," in Power Electronics and Drive Systems (PEDS), 2011 IEEE Ninth International Conference on, 2011, pp. 411-417.

[11] Electric Power Research Institute. Simulation Tool - OpenDSS. Available: http://smartgrid.epri.com/SimulationTool.aspx

[12] R. C. Duggan and T. E. McDermott, "An open source platform for collaborating on smart grid research," in PES General Meeting San Diego, USA,, 2011.

[13] R. Benato, A. Paolucci, R. Turri, "Power flow solution by a complex admittance matrix method," European Transactions on Electrical Power, vol. 11, pp. 181$188,2007$.

[14] ESBNetworks, "Distribution Network: Parameters and Data," K. Sunderland, Ed., ed: ESB Networks, 2011.

[15] M. Albano, R. Turri, S. Dessanti, A. Haddad, H. Griffiths and B. Howat, "Computation of the Electromagnetic Coupling of Parallel Untransposed Power Lines," in Universities Power Engineering Conference, 2006. UPEC '06. Proceedings of the 41st International, 2006, pp. 303-307.

[16] K. M. Sunderland, M. Coppo, M.F. Conlon, R. Turri, "Application of a correction current injection power flow algorithm to an unbalanced 4-wire 
distribution network incorporating TN-C-S earthing," in Power Engineering Conference (UPEC), 2013 48th International Universities', 2013, pp. 1-6.

[17] ESB Networks. (2007, Distribution Code [on-line]. Available: http://www.esb.ie/esbnetworks/en/downloads/Distribution-Code.pdf

[18] W. H. Kersting, Green, R. K.,, "The application of Carson's equation to the steady-state analysis of distribution feeders," in Power Systems Conference and Exposition (PSCE), 2011 IEEE/PES, 2011, pp. 1-6.

[19] EN 50438 Requirements for the connection of micro-generators in parallel with public low-voltage distribution networks, CENELEC, 2007.

[20] K. M. Sunderland, T. Woolmington, J. Blackledge, M.F. Conlon, "Small wind turbines in turbulent (urban) environments: A consideration of normal and Weibull distributions for power prediction," Journal of Wind Engineering and Industrial Aerodynamics, vol. 121, pp. 70-81, 2013.

[21] K. M. Sunderland, G. Mills, M.F. Conlon, "Estimating the wind resource in an urban area: A case study of micro-wind generation potential in Dublin, Ireland," Journal of Wind Engineering and Industrial Aerodynamics, vol. 118, pp. 44-53, 2013.

[22] E. Skoplaki, A.G. Boudouvis, J.A. Palyvo, "A simple correlation for the operating temperature of photovoltaic modules of arbitrary mounting," Solar Energy Materials and Solar Cells, vol. 92, pp. 1393-1402, 2008.

[23] RMDS. (2011, Retail Market Design Service: Standard Load Profiles, . Available: http://www.rmdservice.com/guidance/standard_load_profiles.htm

[24] M. Howley, R. Dennehy, E. Ó Gallachóir, B. Holland, "Energy in Ireland 19902011," Sustainable Energy Authority of Ireland2012.

[25] P. Pillay and M. Manyage, "Definitions of voltage unbalance," IEEE Power Engineering Revie, pp. 50-51, May 2001. 


\section{Figure Captions}

Fig. 1 System representation of the asymmetrical power flow analysis. The network admittance matrix includes shunt elements connected to the external ports (red). Earth connection admittances are also considered as external elements (green).

Fig. 2 Branch element $\pi$-model with the n-phase approach.

Fig. 3 Example of network admittance matrix composition including branches and loads.

Fig. 4 Representation of the power flow solution, using the Y matrix. The currents and potentials are arrays containing the values related to each circuit of the n-phase system.

Fig. 5 Section of (Irish) urban distribution network incorporating load profile variation and domestic (micro) generation connections. The load profile number refers to a seasonal load profile application.

Fig. 6 Transformer, pillar and consumer interconnectivity in context with the pillar/consumer TNC-S earthing provision.

Fig. 7 Load/Generation $(D w G \& D p v G)$ Profiling with 12 noon highlighted for initial analysis. Fig. 8 (a) Pillar/Consumer voltage profiles; prioritising Pillars B and J over the 24hr. period illustrated in Figure 8. (b) Voltage unbalance at pillars.

\section{TABLES}

Table 1 Seasonal load $\left(P_{C o n s}\right)$ and generation $(D w G / D p v G)$ statistical comparison

\begin{tabular}{|c|c|c|c|c|l|}
\hline Spring & \multicolumn{3}{|c|}{ Autumn } & Winter & \multirow{1}{*}{} \\
\cline { 1 - 4 } $\begin{array}{c}P_{\text {Cons }} \\
{[\mathrm{kW}]}\end{array}$ & $\begin{array}{c}P_{\text {Cons }} \\
{[\mathrm{kW}]}\end{array}$ & $\begin{array}{c}P_{D w G} \\
{[\mathrm{~kW}]}\end{array}$ & $\begin{array}{c}P_{D p v G} \\
{[\mathrm{~kW}]}\end{array}$ & $\begin{array}{c}P_{\text {Cons }} \\
{[\mathrm{kW}]}\end{array}$ & \\
\hline 0.85 & 1.01 & 0.91 & 0.79 & 1.23 & $\mathrm{~kW}_{\text {Max }}$ \\
\hline 0.22 & 0.22 & 0.00 & 0.00 & 0.30 & $\mathrm{~kW}_{\text {Min }}$ \\
\hline 0.49 & 0.55 & 0.34 & 0.18 & 0.72 & $\mathrm{~kW}_{\text {Mean }}$ \\
\hline 0.18 & 0.25 & 0.27 & 0.25 & 0.30 & $\mathrm{~kW}_{\text {Std }}$ \\
\hline 11.76 & 13.16 & 17.29 & 8.27 & 4.23 & Energy 24hrs \\
\hline
\end{tabular}


Table 2 Pillar/customer node voltage magnitude (pu) and phase (deg.) results (12pm)

\begin{tabular}{|c|c|c|c|c|c|c|c|}
\hline $\begin{array}{l}\text { Pillar / } \\
\text { Customer }\end{array}$ & $\begin{array}{c}\left|V_{L 1}\right| \\
p u\end{array}$ & $\begin{array}{l}\angle V_{L 1} \\
\text { deg }\end{array}$ & $\begin{array}{c}\left|V_{L 2}\right| \\
p u\end{array}$ & $\begin{array}{c}\angle V_{L 2} \\
\text { deg }\end{array}$ & $\begin{array}{c}\left|V_{L 3}\right| \\
p u\end{array}$ & $\begin{array}{c}\angle V_{L 3} \\
\text { deg }\end{array}$ & $\begin{array}{c}\left|V_{N}\right| \\
p u\end{array}$ \\
\hline B & 1.0487 & -0.04 & 1.0493 & -120.01 & 1.0486 & 119.99 & 0.0002 \\
\hline 1 & & & 1.0493 & -120.01 & & & 0.0002 \\
\hline 2 & & & & & 1.0485 & 119.99 & 0.0003 \\
\hline 3 & & & & & 1.0483 & 119.99 & 0.0002 \\
\hline 4 & & & 1.0493 & -120.01 & & & 0.0007 \\
\hline 5 & 1.0481 & -0.04 & & & & & 0.0007 \\
\hline 6 & 1.0481 & -0.04 & & & & & \\
\hline C & 1.0469 & -0.08 & 1.0483 & -120.03 & 1.0466 & 119.97 & 0.0004 \\
\hline D & 1.0449 & -0.14 & 1.0472 & -120.05 & 1.0444 & 119.95 & 0.0005 \\
\hline $\mathbf{E}$ & 1.0432 & -0.19 & 1.0463 & -120.07 & 1.0425 & 119.93 & 0.0007 \\
\hline $\mathbf{F}$ & 1.0413 & -0.24 & 1.0453 & -120.08 & 1.0402 & 119.91 & 0.0007 \\
\hline G & 1.041 & -0.25 & 1.0452 & -120.09 & 1.0399 & 119.91 & 0.0007 \\
\hline $\mathbf{H}$ & 1.0402 & -0.28 & 1.0448 & -120.09 & 1.0385 & 119.89 & 0.0008 \\
\hline I & 1.0394 & -0.3 & 1.0444 & -120.09 & 1.0373 & 119.87 & 0.0008 \\
\hline $\mathbf{J}$ & 1.0391 & -0.31 & 1.0442 & -120.1 & 1.0369 & 119.87 & 0.0008 \\
\hline 65 & & & & & 1.0363 & 119.87 & 0.0009 \\
\hline 66 & & & 1.0439 & -120.1 & & & 0.0006 \\
\hline 67 & & & & & 1.0365 & 119.87 & 0.0008 \\
\hline 68 & & & 1.0439 & -120.1 & & & 0.0006 \\
\hline 69 & 1.0385 & -0.32 & & & & & 0.0013 \\
\hline 70 & 1.0385 & -0.32 & & & & & 0.0013 \\
\hline 71 & 1.0388 & -0.31 & & & & & 0.0011 \\
\hline 72 & & & & & 1.0366 & 119.87 & 0.0008 \\
\hline 73 & & & 1.0439 & -120.1 & & & 0.0006 \\
\hline 74 & & & 1.0438 & -120.1 & & & 0.0006 \\
\hline
\end{tabular}

Table 3 Pillar voltage magnitude (pu) and phase (deg.) results (12pm): comparison with OpenDSS and DigSilent PowerFactory.

\begin{tabular}{|c|c|c|c|c|c|c|c|c|c|c|c|c|c|c|}
\hline \multirow[b]{2}{*}{ Pillar } & \multicolumn{7}{|c|}{ OpenDSS } & \multicolumn{7}{|c|}{ PowerFactory } \\
\hline & $\begin{array}{c}\left|V_{L 1}\right| \\
p u\end{array}$ & $\begin{array}{c}\angle V_{L 1} \\
\operatorname{deg}\end{array}$ & $\begin{array}{c}\left|V_{L 2}\right| \\
p u\end{array}$ & $\begin{array}{c}\angle V_{L 2} \\
\text { deg }\end{array}$ & $\begin{array}{c}\left|V_{L 3}\right| \\
p u\end{array}$ & $\begin{array}{c}\angle V_{L 3} \\
\text { deg }\end{array}$ & $\begin{array}{c}\left|V_{N}\right| \\
p u\end{array}$ & $\begin{array}{c}\left|V_{L 1}\right| \\
p u\end{array}$ & $\begin{array}{c}\angle V_{L 1} \\
\operatorname{deg}\end{array}$ & $\begin{array}{c}\left|V_{L 2}\right| \\
p u\end{array}$ & $\begin{array}{c}\angle V_{L 2} \\
\text { deg }\end{array}$ & $\begin{array}{c}\left|V_{L 3}\right| \\
p u\end{array}$ & $\begin{array}{c}\angle V_{L 3} \\
\text { deg }\end{array}$ & $\begin{array}{c}\left|V_{N}\right| \\
p u\end{array}$ \\
\hline B & 1.0487 & 0 & 1.0494 & -120 & 1.0486 & 120 & 0.0002 & 1.0487 & 0.0 & 1.0493 & -120.0 & 1.0486 & 120.0 & 0.0002 \\
\hline $\mathrm{C}$ & 1.0469 & -0.1 & 1.0484 & -120 & 1.0467 & 120 & 0.0003 & 1.0469 & -0.1 & 1.0483 & -120.0 & 1.0467 & 120.0 & 0.0004 \\
\hline D & 1.045 & -0.1 & 1.0472 & -120.1 & 1.0445 & 119.9 & 0.0005 & 1.0450 & -0.1 & 1.0472 & -120.1 & 1.0444 & 119.9 & 0.0005 \\
\hline $\mathbf{E}$ & 1.0433 & -0.2 & 1.0463 & -120.1 & 1.0426 & 119.9 & 0.0006 & 1.0433 & -0.2 & 1.0462 & -120.1 & 1.0426 & 119.9 & 0.0007 \\
\hline $\mathbf{F}$ & 1.0415 & -0.2 & 1.0454 & -120.1 & 1.0403 & 119.9 & 0.0007 & 1.0414 & -0.2 & 1.0453 & -120.1 & 1.0403 & 119.9 & 0.0008 \\
\hline G & 1.0412 & -0.3 & 1.0453 & -120.1 & 1.04 & & 0.0007 & 1.0412 & -0.3 & 1.0452 & -120.1 & 1.0400 & 119.9 & 0.0007 \\
\hline $\mathbf{H}$ & 1.0404 & -0.3 & 1.0449 & -120.1 & 1.0387 & 119.9 & 0.0008 & 1.0403 & -0.3 & 1.0448 & -120.1 & 1.0387 & 119.9 & 0.0008 \\
\hline $\mathbf{I}$ & 1.0396 & -0.3 & 1.0445 & -120.1 & 1.0375 & 119.9 & 0.0008 & 1.0396 & -0.3 & 1.0443 & -120.1 & 1.0375 & 119.9 & 0.0008 \\
\hline $\mathbf{J}$ & 1.0393 & -0.3 & 1.0443 & -120.1 & 1.0371 & 119.9 & 0.0008 & 1.0392 & -0.3 & 1.0441 & -120.1 & 1.0370 & 119.9 & 0.0008 \\
\hline
\end{tabular}




\section{FIGURES}

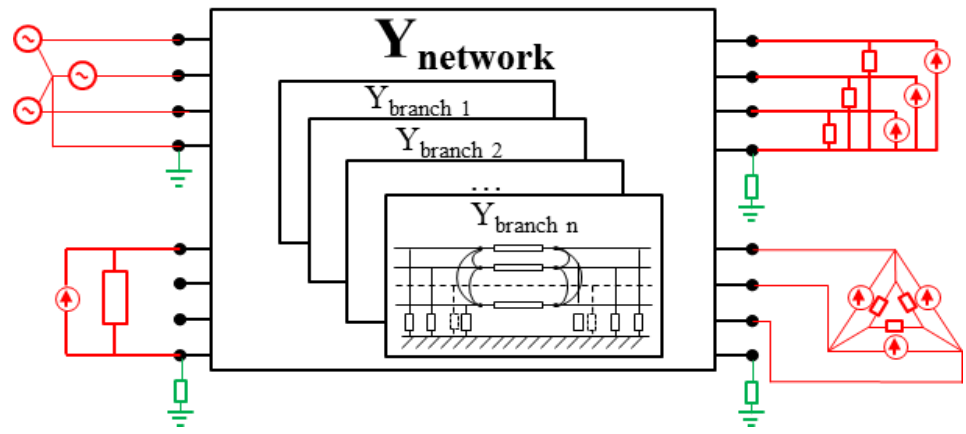

Figure 1

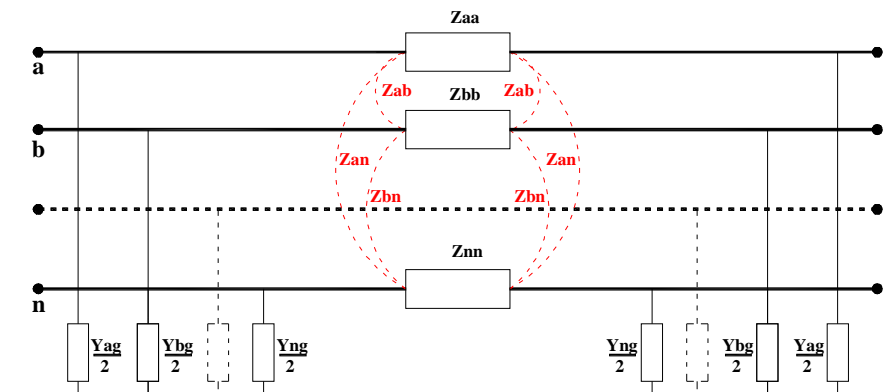

Figure 2

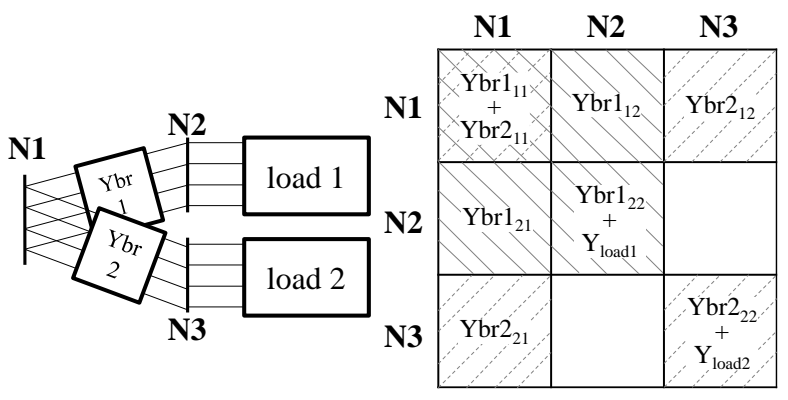

Figure 3

$$
\left[\begin{array}{c}
\mathbf{I}_{\mathbf{S L}}{ }_{a \ldots n} \\
\hdashline \mathbf{\Delta I}_{\mathbf{I}_{a \ldots n}} \\
\vdots \\
\vdots \\
\mathbf{\Delta I}_{m_{a \ldots n}}
\end{array}\right]=\left[\begin{array}{c:c}
\mathbf{Y}_{\mathbf{S L}-\mathbf{S L}} & \mathbf{Y}_{\mathbf{S L}-\mathbf{S H}} \\
\hdashline & \\
\mathbf{Y}_{\mathbf{S H}-\mathbf{S L}} & \mathbf{Y}_{\mathbf{S H}-\mathbf{S H}} \\
& \\
&
\end{array}\right]\left[\begin{array}{c}
\mathbf{E}_{\mathbf{S L}} \ldots \ldots n \\
\hdashline \mathbf{E}_{2_{a \ldots n}} \\
\vdots \\
\vdots \\
\mathbf{E}_{m_{a \ldots n}}
\end{array}\right]
$$

Figure 4 


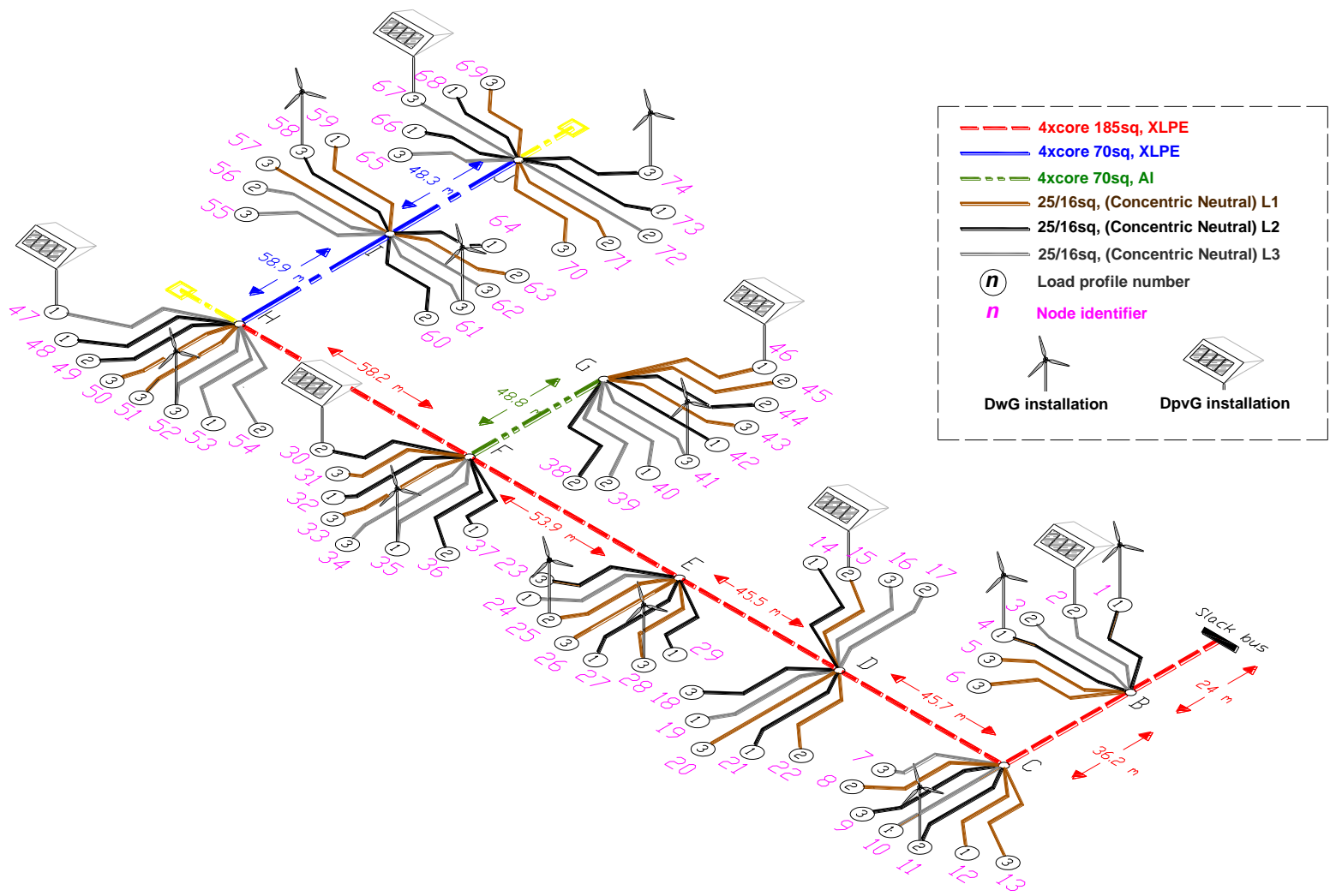

Figure 5

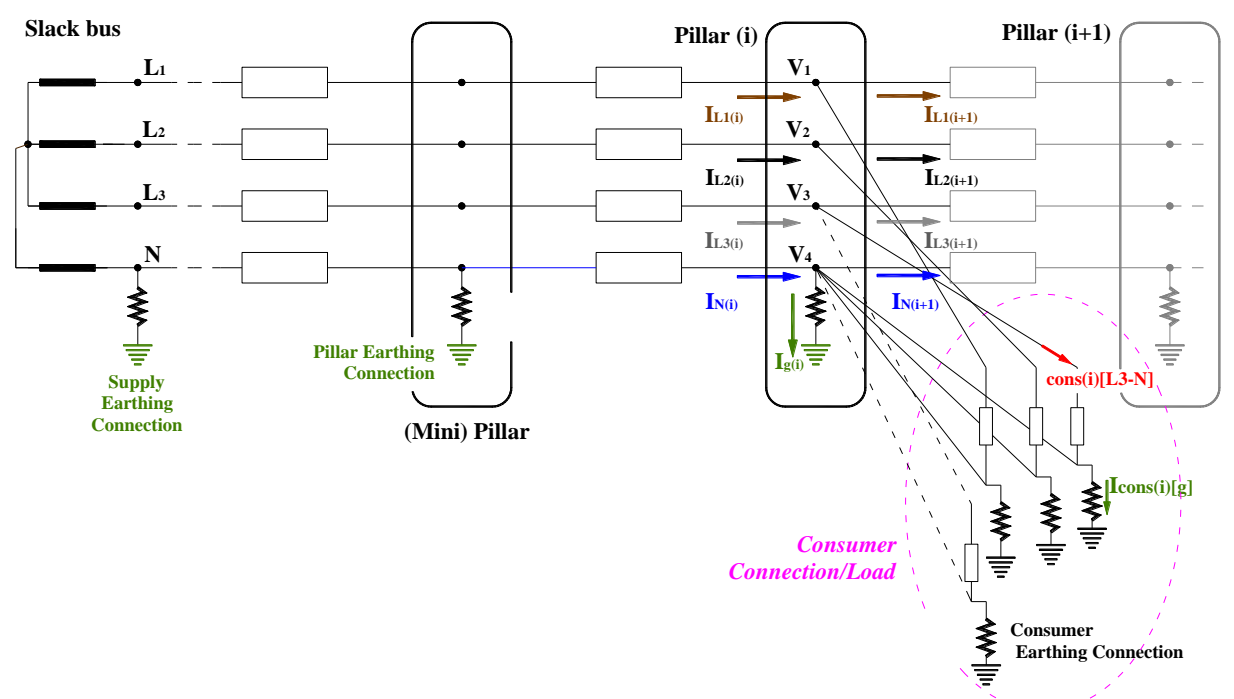

Figure 6 


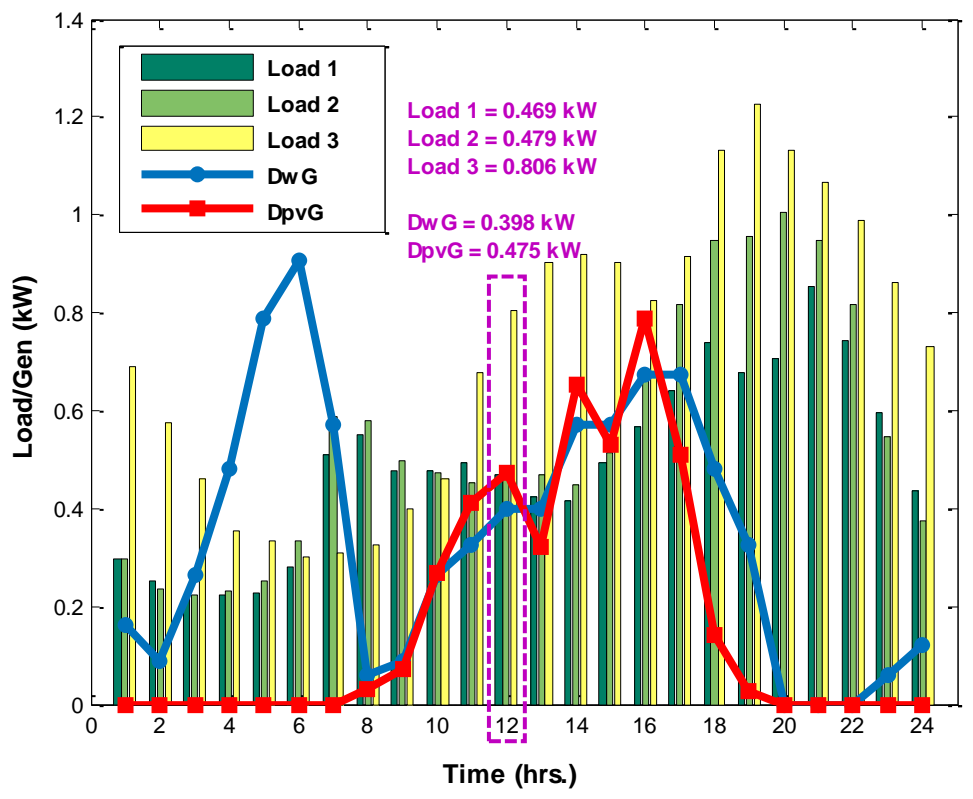

Figure 7

(a)
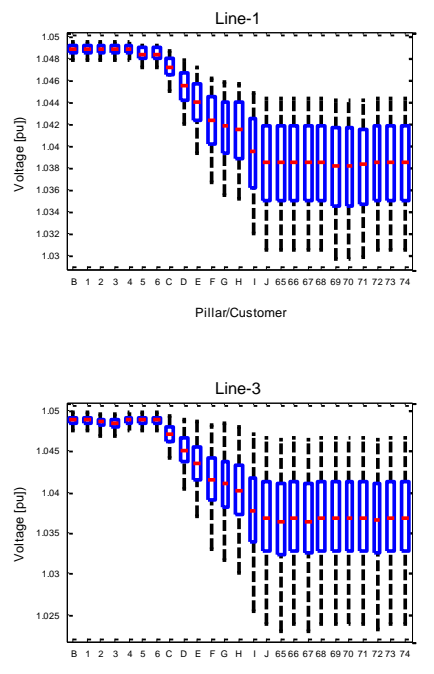
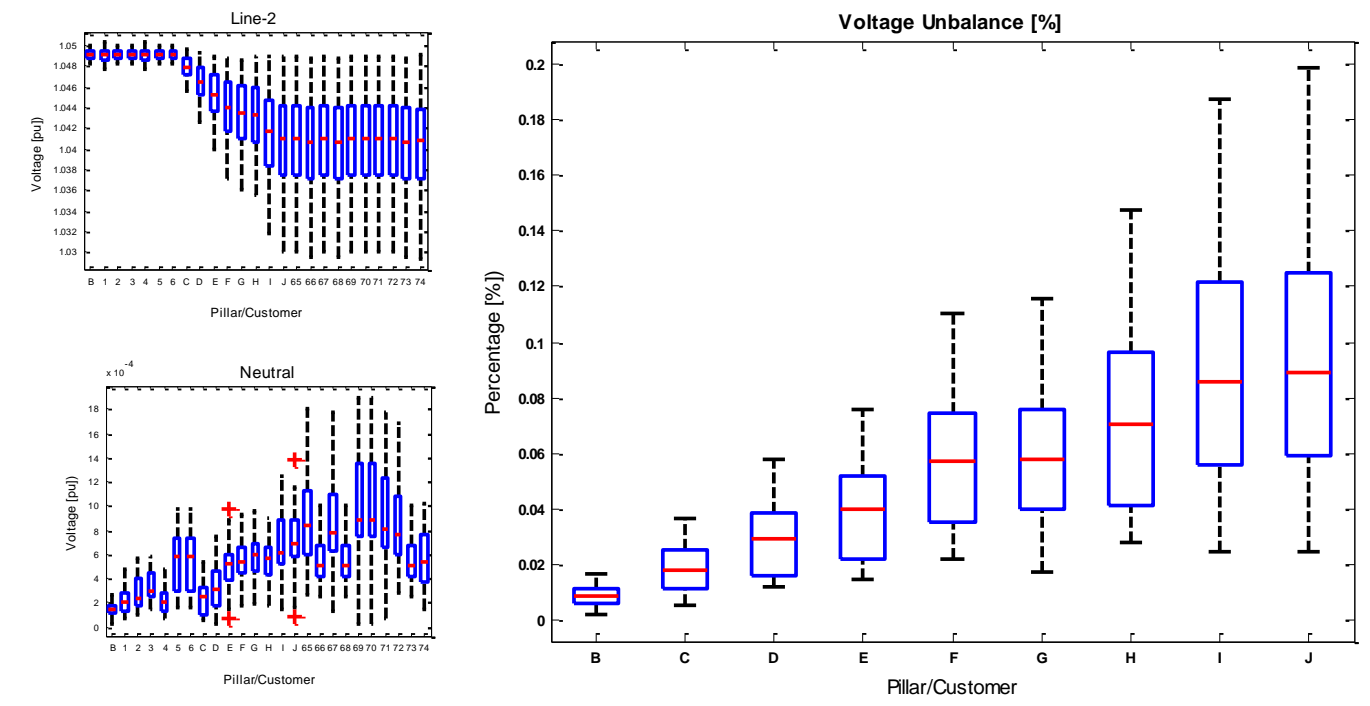

Figure 8 